\title{
Pathwise optimality for benchmark tracking
}

\author{
Paolo Dai Pra \\ Wolfgang J. Runggaldier \\ Marco Tolotti \\ Dipartimento di Matematica Pura ed Applicata \\ Universitá di Padova, Via Belzoni 7 \\ 35131 - Padova, Italy \\ e-mail: daipra,runggal@math.unipd.it,mtolotti@hotmail.com
}

\begin{abstract}
We consider the problem of investing in a portfolio in order to track or "beat" a given benchmark. We study this problem from the point of view of almost sure/pathwise optimality. We first obtain a control that is optimal in the mean and this control is then shown to be also pathwise optimal. The standard Merton model leads to lognormality of the value process so that it does not possess the required ergodic properties. We obtain ergodicity by transforming the process so that it remains bounded thereby using a method that can be related to a random time change. We furthermore describe a general approach to solve the HJB equation corresponding to the given problem setup.
\end{abstract}

Keywords: Optimal portfolios, benchmark tracking, optimal stochastic control, pathwise optimality, solutions of HJB equations, random time change.

\section{INTRODUCTION}

We consider a problem from institutional money management, where the objective of the investor/money manager is that of tracking or, better, outperforming a given stochastic benchmark; the benchmark may be an index process such as the S\&P 500 index, it may however also represent other economic quantities such as the value process of a non traded asset like the inflation or the exchange rate. Typically we shall assume that the investor may invest in a certain number of risky assets in addition to a non risky one and let $X_{t}^{\pi}$ denote the investor's wealth at time $t$ corresponding to an investment strategy $\pi$. The benchmark process will be denoted by $Y_{t}$ and we shall make the realistic assumption that it is not perfectly correlated with the investment opportunities so that the investor cannot completely control his risk (the market is incomplete).

This portfolio problem, that is sometimes also called active portfolio management, has been studied by various authors and here we refer in particular to [2]. As in [2] we shall consider as relevant state variable the ratio $Z_{t}^{\pi}:=X_{t}^{\pi} Y_{t}^{-1}$ of the investor's wealth to the benchmark. A natural way to proceed (see again [2]) is then to consider the process $Z_{t}^{\pi}$ up to the exit from a bounded domain and choose as objective the minimization of the discounted expected loss that penalizes the deviation of $Z_{t}^{\pi}$ from the constant 1 in the case of "benchmark tracking" and the amount by which $Z_{t}^{\pi}$ falls below 1 in the case when the objective is that of "beating the benchmark".

While the classical criteria such as the one described above are criteria in the mean, namely they 
involve expected values of costs/losses, in this paper we aim at a stronger form of criterion, more precisely that of a.s.-optimality (see e.g. [8], [7], [6], [9]) that may in fact be quite appropriate for benchmark tracking/beating. The a.s.-optimality criteria in use concern an infinite horizon, which may still make sense in economic/financial applications every time an investor makes his plans over a long horizon. To keep matters simple in this first attempt to apply an a.s.criterion to an investment problem, we shall consider here only symmetric cost functions, i.e. we shall only consider the benchmark tracking problem, thereby penalizing symmetrically both over- and undershoots of $Z_{t}^{\pi}$ with respect to 1 . An economically more meaningful asymmetric cost function that penalizes only undershoots/shortfalls and corresponds to the typical benchmark beating/outperforming can still be dealt with in our approach, but at the expense of less analytical tractability.

More precisely, the aim is to find an investment strategy $\pi^{*}$ such that for the corresponding ratio process we have $Z_{t}^{\pi^{*}} \approx 1$. Define

$$
J_{T}(\pi):=\int_{0}^{T} c\left(Z_{t}^{\pi}\right) d t
$$

where, using a penalization by the quadratic deviation of $Z_{t}^{\pi}$ from 1 , one would take $c(z)=(z-1)^{2}$. Denoting by $P^{\pi}$ the measure induced by the investment strategy $\pi$ (being interested in an infinite horizon, we shall not make explicit the dependence on the initial value of $Z_{t}^{\pi}$ ), the criterion of almost sure optimality/pathwise optimality is roughly as follows (for a more detailed discussion see subsection II-B below). Given strategies $\pi$ and $\pi^{*}$, let $P^{\pi^{*}, \pi}$ be a measure having $P^{\pi^{*}}$ and $P^{\pi}$ as marginals and let $g:[0, \infty) \rightarrow[0, \infty)$ be a nondecreasing function with $\lim _{T \rightarrow \infty} g(T)=0$. The control strategy $\pi^{*}$ is then said to be a.s. $g$-optimal if, for all admissible $\pi$ and all coupling measures $P^{\pi^{*}, \pi}$, one has that

$\lim _{T \rightarrow \infty} g(T)\left[J_{T}\left(\pi^{*}\right)-J_{T}(\pi)\right]^{+}=0, \quad P^{\pi^{*}, \pi}-$ a.s. where $[\cdot]^{+}$denotes the positive part of its argument.

The standard way of approaching a problem with the criterion of a.s. $g$-optimality is (see [9]) to consider a corresponding criterion of optimality in the mean, which in our case becomes

$$
\lambda^{\pi}=\limsup _{T \rightarrow \infty} \frac{1}{T} E^{\pi}\left\{\int_{0}^{T} c\left(Z_{t}^{\pi}\right) d t\right\} \longrightarrow \min
$$

and to investigate when the mean-optimal control is also a.s./pathwise optimal and this will also be our approach here.

For the standard market models, namely those that that go back to Merton ([5]), the price processes are geometric Brownian motions (lognormal processes) so that already for simple strategies $\pi$ the value of $\lambda^{\pi}$ in (3) above becomes infinite. Here comes now our main methodological contribution in this paper : we modify the dynamics of the process $Z_{t}^{\pi}$ so that it becomes a.s. bounded (in a large domain) and still maintains the main characteristics corresponding to lognormal models. Given our ergodic criterion, this cannot be accomplished by simply stopping the process upon exit from a given compact set. Here we change instead suitably the drift and diffusion coefficients, which under certain assumptions can also be interpreted as a random time change and by which the controlled process is increasingly slowed down as it gets closer and closer to a given boundary. An interesting aspect that turns out in this context is that the optimal control and the solution of the Hamilton-Jacobi-Bellman (HJB) equation do not depend on the particular choice of the random time change. With the thus modified process $Z_{t}^{\pi}$ we shall not only obtain the right ergodic behavior in order to make the mean-optimality criterion (3) meaningful, but it will furthermore allow us to show that the mean-optimal control is also a.s. $g$-optimal.

A final methodological aspect of this paper concerns the problem of solving the HJB equation associated to the given (infinite-horizon) stochastic control problem with the criterion of optimality in the 
mean. More precisely, denoting by $\mathcal{L}^{\pi}$ the generator of the process $Z_{t}^{\pi}$ that is controlled by the strategy $\pi_{t}$ and letting $c(z)$ be our instantaneous cost function that we assume to be bounded from below (e.g. $\left.c(z)=(z-1)^{2}\right)$, the infinite-horizon criterion of optimality in the mean leads to solving (see section III-A below)

$$
\lambda=\inf _{\pi}\left[\mathcal{L}^{\pi} \phi(z)+c(z)\right]=\mathcal{L}^{\pi^{*}} \phi(z)+c(z)
$$

for $\pi^{*}, \phi(\cdot)$ and $\lambda$. Assuming that an optimal minimizing $\pi^{*}$ can be found, the remaining problem is then to find, for given $\mathcal{L}^{\pi}$ and $c$, the pair $\lambda$ and $\phi(z)$. The traditional way is to guess (make an "Ansatz" for) a possible function $\phi(\cdot)$ which works only in specific cases. Since a same objective may also be reached by using analytically different cost functions, we shall generalize the problem of solving the HJB equation (4) by considering a class $c^{\epsilon, K}(z)$ of possible cost functions and, given the dynamics of the controlled process, i.e. given its generator $\mathcal{L}^{\pi}$, search for a triple $\left(\lambda, \phi, c^{\epsilon, K}\right)$ such that (4) holds.

The paper is structured as follows. In the next section II we describe more precisely our problem setup with the criteria of optimality in the mean and almost sure/pathwise optimality (subsection IIA). In subsection II-B we then present in more detail the criterion of pathwise optimality recalling a result that shows under what conditions a control that is optimal in the mean is also almost surely optimal. Subsection II-C concerns the bounding of the controlled state process by a method that can be related to a random time change. Section III is mainly dedicated to determine explicitly a control that is optimal in the mean (subsection III-A) and for which in subsection III-B it is then shown that it is also almost sure/pathwise optimal. Subsection III-A is divided further into subsubsections. In particular, in III-A.1 we solve an auxiliary HJB equation without the time change and for polynomial $c(z)$. In III-A.2 it is then shown how the solution for the auxiliary HJB can be transformed into a solution of the actual
HJB equation.

\section{MODEL AND PROBLEM FORMULATION}

\section{A. The basic setup}

We consider a market with a nonrisky asset and a number of risky assets. The price $B_{t}$ of the nonrisky asset evolves according to

$$
d B_{t}=r B_{t} d t
$$

where $r$ is a fixed spot rate of interest. Let $k$ denote the number of risky assets. Given a filtered and complete probability space $\left(\Omega, \mathcal{F}, \mathcal{F}_{t}, P\right)$ with $P$ the "real world probability measure", the prices $S_{t}^{i}, i=$ $1, \cdots, k$ of the risky assets are supposed to evolve according to a standard lognormal model, namely

$$
d S_{t}^{i}=\mu^{i} S_{t}^{i} d t+\sum_{j=1}^{k} \sigma^{i j} S_{t}^{i} d w_{t}^{j} \quad(i, j=1, \cdots, k)
$$

with given drift coefficients $\mu^{i}$ and a given volatility matrix $\sigma=\left\{\sigma^{i j}\right\}_{i, j=1, \cdots, k}$ that is supposed to be an invertible matrix and where $w_{t}=\left(w_{t}^{1}, \cdots, w_{t}^{k}\right)$ is a $\left(P, \mathcal{F}_{t}\right)$-Wiener process.

Consider as investment strategy an $\mathcal{F}_{t}-$ predictable process

$$
\varphi_{t}=\left(\varphi_{t}^{0}, \varphi_{t}^{1}, \cdots, \varphi_{t}^{k}\right)
$$

where $\varphi_{t}^{0}$ denotes the number of units of the nonrisky asset that are kept in the portfolio at time $t$ and, analogously, $\varphi_{t}^{i}$ is the number of units of the $i$-th asset. The value process corresponding to this strategy is then

$$
X_{t}^{\varphi}=\varphi_{t}^{0} B_{t}+\sum_{i=1}^{k} \varphi_{t}^{i} S_{t}^{i}
$$

and the strategy $\varphi_{t}$ is said to be self financing if

$$
d X_{t}^{\varphi}=\varphi_{t}^{0} d B_{t}+\sum_{i=1}^{k} \varphi_{t}^{i} d S_{t}^{i}
$$

It is convenient to represent investment strategies in terms of the fraction of wealth that is invested in the 
individual assets rather than in terms of number of units. Putting then

$$
\pi_{t}^{i}:=\frac{\varphi_{t}^{i} S_{t}^{i}}{X_{t}^{\varphi}}, i=1, \cdots, k ; \quad 1-\sum_{i=1}^{k} \pi_{t}^{i}=\frac{\varphi_{t}^{0} B_{t}}{X_{t}^{\varphi}}
$$

the wealth process of a self financing strategy, which we now indicate by $X_{t}^{\pi}$, can then be represented as

$$
\begin{aligned}
d X_{t}^{\pi}=X_{t}^{\pi} & \left(r+\sum_{i=1}^{k} \pi_{t}^{i}\left(\mu^{i}-r\right)\right) d t \\
& +X_{t}^{\pi} \sum_{i=1}^{k} \sum_{j=1}^{k} \pi_{t}^{i} \sigma^{i j} d w_{t}^{j}
\end{aligned}
$$

With obvious meaning of the symbols we may rewrite (11) in vector notation as

$$
d X_{t}^{\pi}=X_{t}^{\pi}\left[\left(r+\pi_{t}^{\prime}(\mu-r \underline{1})\right) d t+\pi_{t}^{\prime} \sigma d w_{t}\right] .
$$

In what follows we restrict ourselves to strategies $\pi$ such that (12) has a weak, non-exploding solution. A precise definition of admissible strategies requires, however, a further discussion that we postpone to subsection 2.3.

Next we consider a benchmark (index) process that we shall view as the value process of a theoretical portfolio consisting of a large number of assets. Denoting this value process by $Y_{t}$, a standard way to model its evolution is, by analogy to (12), the following

$$
d Y_{t}=Y_{t}\left[a d t+b^{\prime} d w_{t}+\beta d v_{t}\right]
$$

where $v_{t}$ is a scalar $\left(P, \mathcal{F}_{t}\right)$-Wiener process, independent of $w_{t}$. Since the portfolio $Y_{t}$ includes also assets beyond those with prices $S_{t}^{i}$ that make up the actual portfolio with value $X_{t}^{\pi}$, this $v_{t}$ synthetizes the Wiener processes that drive these additional assets and are not included in $w_{t}$.

The benchmark tracking problem now consists in choosing $\pi_{t}^{*}$ such that, possibly, $X_{t}^{\pi^{*}} \approx Y_{t}$. Problems of this kind have to some extent already been studied in the literature and here we refer in particular to [2]. As in [2] define the ratio process
$Z_{t}^{\pi}:=X_{t}^{\pi} Y_{t}^{-1}$ that, given (12), (13) satisfies by Ito's rule

$$
\begin{aligned}
d Z_{t}^{\pi}=Z_{t}^{\pi}(\hat{r} & \left.+\pi_{t}^{\prime} \hat{\mu}\right) d t \\
& +Z_{t}^{\pi}\left(\pi_{t}^{\prime} \sigma-b^{\prime}\right) d w_{t}-Z_{t}^{\pi} \beta d v_{t}
\end{aligned}
$$

where we have put

$$
\hat{r}=r-a+b^{\prime} b+\beta^{2} \quad, \quad \hat{\mu}=\mu-r \underline{1}-\sigma b
$$

The problem is now to choose $\pi_{t}^{*}$ so that, possibly, $Z_{t}^{\pi^{*}} \approx 1$. One may take as natural instantaneous cost function (see Proposition 3.8 and Remark 3.9 below for comments of this choice)

$$
c\left(z_{t}, \pi_{t}\right)=c\left(z_{t}\right)=\left(z_{t}-1\right)^{2}
$$

and let $J_{T}(\pi)$ be as in (1). Given $\epsilon$ and $K$ with $0<\epsilon<K<\infty$, consider as acceptable values for $Z_{t}^{\pi}$ those for which $Z_{t}^{\pi} \in[\epsilon, K]$ (to this effect soo also Remark 2.1) and, given a control $\pi$, define $\tau=\inf \left\{t \geq 0: Z_{t}^{\pi} \notin[\epsilon, K]\right\}$. Then, one aims at minimizing the cost functional

$$
\mu^{\pi}=E^{\pi}\left\{J_{\tau}(\pi)\right\}
$$

This optimal control problem has been solved in [2]; it turns out that the optimal control $\pi^{*}$ is a nonlinear feedback in $Z_{t}$ and, as will be seen below, it does not depend on the choice of $\epsilon$ and $K$.

One purpose of this paper is to show that the optimal control $\pi^{*}$ has stronger optimality properties than just optimality in the mean. To this end we use the theory of pathwise optimality, as developed in [9], and that we will briefly summarize in next subsection. This theory applies to models for which the ergodic cost functional

$$
\lambda^{\pi}=\limsup _{T \rightarrow \infty} \frac{1}{T} E^{\pi}\left\{J_{T}(\pi)\right\}
$$

is finite for a reasonable class of strategies. A glance at equation (14) shows that this is not the case in our model. If we choose e.g. a constant strategy $\pi_{t} \equiv$ const., then $Z_{t}$ is a lognormal process; the second moment of $Z_{t}$ grows exponentially fast in time, and therefore $\lambda^{\pi}=+\infty$. 
Our approach may be considered as alternative to the one leading to the cost functional (17). Rather than stopping the process when it reaches the boundary of $[\epsilon, K]$, we perform a transformation that in a sense is equivalent to a random time change and whose effect is, roughly speaking, to speed up the time scale (equivalent to slowing down the process) as the process gets close to the boundary of $[\epsilon, K]$. The controlled process $\tilde{Z}_{t}$ that is obtained via this transformation has the following features:

- It takes values, with probability one and for all $t \geq 0$, in the interval $[\epsilon, K]$. The ergodic cost functional (18) is, therefore, finite.

- The optimal control $\pi^{*}$, as a feedback in $\tilde{Z}$, is the same as for the cost functional (17). In particular, at least at a formal level, all reasonable time changes lead to the same optimal feedback.

- In the new time scale the control, which is optimal for the ergodic cost criterion (18), satisfies the assumptions for pathwise optimality.

\section{Remark 2.1:}

i) The above considerations show that, in order to obtain a solution with the stronger property of pathwise optimality, one has to restrict the evolution of $Z_{t}^{\pi}$ to a compact set and in such a way that this evolution has the required ergodic properties. The choice of the compact set $[\epsilon, K]$ is arbitrary. It does not affect the optimal strategy, but affects the cost criterion and thus also the optimal value of the cost. The modified cost criterion has however the same effect as the original criterion, namely to keep the wealth process as close as possible to the benchmark in the sense of the quadratic deviation (a same objective can in fact be achieved by means of different cost functions).

ii) From the applied financial point of view the restriction of $Z_{t}^{\pi}$ to $[\epsilon, K]$ can be interpreted as restricting the wealth process to a band around the benchmark, where the width of this band depends on the magnitude of the benchmark, namely $X_{t}^{\pi} \in\left[\epsilon Y_{t}, K Y_{t}\right]$. This is financially meaningful in the sense that no investment manager will be allowed to choose a strategy that lets his portfolio deviate too far from the benchmark; within this class of strategies he will then choose the one that comes closest to the benchmark.

We shall call optimal in the mean a solution of the optimal control problem with the ergodic cost functional (18). A stronger form of optimality will be introduced in the next section.

\section{B. Pathwise otimality}

In this section we give a short introduction to pathwise optimality.

Given a measurable space $U$, measurable functions $f: \mathbb{R}^{k} \times U \rightarrow \mathbb{R}^{k}, \sigma: \mathbb{R}^{k} \times U \rightarrow \mathbb{R}^{k \times k^{\prime}}$ and a $k^{\prime}$-dimensional Brownian motion $w_{t}$, consider the controlled stochastic differential equation

$$
\begin{aligned}
d x_{t} & =f\left(x_{t}, u_{t}\right) d t+\sigma\left(x_{t}, u_{t}\right) d w_{t}, \\
x_{0} & \sim \mu,
\end{aligned}
$$

where $\mu$ is a probability on $\mathbb{R}^{k}$. This equation is naturally associated to the family of operators

$$
\mathcal{L}^{u}=\frac{1}{2} \sum_{i, j=1}^{k} a_{i j}(x, u) \frac{\partial^{2}}{\partial x_{i} \partial x_{j}}+\sum_{i=1}^{k} f_{i}(x, u) \frac{\partial}{\partial x_{i}},
$$

with $a(x, u)=\sigma^{\prime}(x, u) \sigma(x, u)$.

Suppose we fix a nonnegative, measurable function $c: \mathbb{R}^{k} \times U \rightarrow[0,+\infty)$. We also denote by $\mathcal{C}$ the function space $\mathcal{C}\left([0,+\infty), \mathbb{R}^{k}\right)$, endowed with the topology of uniform convergence on compact sets, and the associated Borel $\sigma$-field. In this section, we let $\left(\mathcal{F}_{t}\right)_{t \geq 0}$ be the natural filtration in $\mathcal{C}$.

Definition 2.2: A progressively measurable function $u:[0,+\infty) \times \mathcal{C} \rightarrow U$ is said to be a control if the following conditions hold.

1) There exists a probability measure $P^{u}$ on $\mathcal{C}$ such that for every $f \in \mathcal{C}^{2}$ the process

$$
z_{t}=f\left(x_{t}\right)-\int_{0}^{t}\left(\mathcal{L}^{u_{s}} f\right)\left(x_{s}\right) d s
$$


is a $P^{u}$-local martingale, and $P^{u} \circ \Pi_{0}^{-1}=\mu$.

2) For all $t \geq 0$ we have

$$
J_{t}(u)=\int_{0}^{t} c\left(x_{s}, u_{s}\right) d s<+\infty \text { a.s. }
$$

Depending on the circumstances, one may restrict oneself to a suitable subset $\mathcal{U}$ of the set of all controls. Elements of $\mathcal{U}$ will be called admissible controls. Consider a nondecreasing function $g$ : $[0,+\infty) \rightarrow(0,+\infty)$ such that $\lim _{T \rightarrow+\infty} g(T)=0$.

Definition 2.3: We say that a control $u^{*} \in \mathcal{U}$ is $g$-optimal a.s. (respectively, in probability) if for all $u \in \mathcal{U}$ and for all probability measures $P^{u, u^{*}}$ on $\mathcal{C} \times \mathcal{C}$ having marginals $P^{u}$ and $P^{u^{*}}$ and such that

$$
P^{u, u^{*}}\left\{(x, y) \in \mathcal{C} \times \mathcal{C}: x_{0} \neq y_{0}\right\}=0
$$

we have

$$
\lim _{T \rightarrow+\infty} g(T)\left[J_{T}\left(u^{*}\right)-J_{T}(u)\right]^{+}=0 \quad P^{u, u^{*} \text { a.s. }}
$$

(respectively, in probability with respect to $P^{u, u^{*}}$ ).

Consider the stationary Hamilton - Jacobi Bellman equation (SHJB)

$$
\inf _{u \in U}\left[\mathcal{L}^{u} \phi(x)+c(x, u)\right]=\lambda,
$$

in the unknowns $(\phi, \lambda)$. We are now ready to state the basic result on pathwise optimality (see [9]).

Theorem 2.4: Suppose the following conditions hold:

(i) There exists a solution $(\phi, \lambda)$ of (SHJB), with $\phi \in \mathcal{C}^{2}\left(\mathbb{R}^{d}\right)$. The "inf" in (SHJB) is attained at $v=k(x)$, and the feedback $u_{t}^{*}=k\left(x_{t}\right)$ is an admissible control. Moreover, for each admissible control $u$, the processes $\phi\left(x_{t}\right)$ and $\frac{\partial \phi}{\partial x}\left(x_{t}\right) \sigma\left(x_{t}, u_{t}\right)$ are bounded $P^{u}$-a.s.

(ii) There exists a constant $C>0$ and $m>0$ such that

$$
E^{P^{u^{*}}}\left\{\left[\int_{0}^{T} c\left(x_{t}, u_{t}\right) d t\right]^{m}\right\} \leq C T^{m}
$$

for all $T>0$.

(iii) Defining $q(t)=t^{1 / 2} g(t)$, and letting $m$ be the same constant as in (ii), one has

$$
\int_{1}^{+\infty} q^{m}(t) d t<+\infty
$$

Then the feedback $u^{*}$ is $g$-optimal a.s.

Remark 2.5: For systems whose state variable takes values in a compact set, the boundedness condition for $\phi\left(x_{t}\right)$ is easily satisfied. This is the case for the model to which Theorem 2.4 will be applied. Boundedness of $\frac{\partial \phi}{\partial x}\left(x_{t}\right) \sigma\left(x_{t}, u_{t}\right)$, in the case $\sigma(x, u)$ depends on $u$, is more severe if one admits unbounded controls. This conditions can actually be weakened (see [9]): the conclusions of Theorem 2.4 hold if we replace boundedness of $\frac{\partial \phi}{\partial x}\left(x_{t}\right) \sigma\left(x_{t}, u_{t}\right)$ by

$$
\left\|\frac{\partial \phi}{\partial x}\left(x_{t}\right) \sigma\left(x_{t}, u_{t}\right)\right\|^{2} \leq C\left[c\left(x_{t}, u_{t}\right)+D\right] \text { a.s. }
$$

for some $C, D>0$. Condition (26), however, does not help if the instantaneous cost $c(\cdot, \cdot)$ is independent of $u$, as it happens for the cost in (16). For this reason we will later assume that admissible controls are a.s. bounded.

\section{Bounded controlled processes (random time change)}

We now illustrate the method to transform the process $Z_{t}$ in (14) into a process that takes values in $[\epsilon, K]$ at all positive times. This method corresponds to a random time change, and its justification was discussed in Remark 2.1.

Actually, we shall start out by defining directly a suitable random time change. For this purpose, given a strategy $\pi$, let $Z_{t}^{\pi}$ be the process according to (14). Fix an interval $[\epsilon, K]$ and a continuous function $\gamma: \mathbb{R} \rightarrow[0,+\infty)$ such that $\gamma(z)>0$ for $z \in(\epsilon, K)$, and $\gamma(z) \equiv 0$ for $z \notin(\epsilon, K)$ (for a specific choice of such a function $\gamma(\cdot)$ see Theorem 3.6 below). As in section II-A we let, for given $\pi, \tau=\inf \{t \geq 0$ : $\left.Z_{t}^{\pi} \notin[\epsilon, K]\right\}$. Assuming that, with probability one, $Z_{0}^{\pi} \in(\epsilon, K)$, define, for $t<\tau$

$$
\alpha(t)=\int_{0}^{t} \frac{1}{\gamma^{2}\left(Z_{s}^{\pi}\right)} d s .
$$

$\alpha$ is a (random), continuous, strictly increasing function on $[0, \tau)$, thus it admits a continuous inverse 
$\alpha^{-1}$. The crucial point consists in choosing $\gamma$ so that

$$
\lim _{t \uparrow \tau} \alpha(t)=+\infty \quad \text { a.s. }
$$

which implies that the domain of $\alpha^{-1}$ is $[0,+\infty)$. Under condition (28), the process

$$
Z_{t}^{\pi, \gamma}=Z_{\alpha^{-1}(t)}^{\pi}
$$

is defined for all $t \geq 0$, and takes values in $(\epsilon, K)$. Moreover, it is not hard to show (see e.g. [1], Chapter 5) that

$$
\begin{aligned}
d Z_{t}^{\pi, \gamma}= & \gamma^{2}\left(Z_{t}^{\pi, \gamma}\right) Z_{t}^{\pi, \gamma}\left(\hat{r}+\pi_{t}^{\prime} \hat{\mu}\right) d t \\
& +\gamma\left(Z_{t}^{\pi, \gamma}\right) Z_{t}^{\pi, \gamma}\left(\pi_{t}^{\prime} \sigma-b^{\prime}\right) d w_{t} \\
& -\gamma\left(Z_{t}^{\pi, \gamma}\right) Z_{t}^{\pi} \beta d v_{t}
\end{aligned}
$$

In terms of formal computations, this means that, under the time change $t \rightarrow \alpha^{-1}(t)$, we have $d t \rightarrow \gamma^{2}\left(Z_{t}^{\pi, \gamma}\right) d t, d w_{t} \rightarrow \gamma\left(Z_{t}^{\pi, \gamma}\right) d w_{t}, d v_{t} \rightarrow$ $\gamma\left(Z_{t}^{\pi, \gamma}\right) d v_{t}$. The associated cost functional must be modified accordingly:

$$
\begin{aligned}
J_{T}(\pi) & =\int_{0}^{T} c\left(Z_{t}^{\pi}\right) d t \rightarrow J_{T}^{\gamma}(\pi) \\
& =\int_{0}^{T} \gamma^{2}\left(Z_{t}^{\pi, \gamma}\right) c\left(Z_{t}^{\pi, \gamma}\right) d t .
\end{aligned}
$$

For reasons that will become clear in section III-A. 2 below, it will be convenient to consider, instead of a fixed instantaneous cost function $c(z)$, the entire family of its translates $c(z)-\Lambda$. Here we simply point out that this translation of the instantaneous cost produces a simple translation of $J_{T}$, but $J_{T}^{\gamma}$ is not transformed in a simple way. Thus, after the time change, the control problem with the translated instantaneous cost is not necessarily equivalent to the one without translation (the optimal control law will however be seen to be independent of this translation).

Condition (28), that is essential for the random time change to make sense, is a rather delicate one. It involves relations between the behavior of $\gamma$ near the boundary of $[\epsilon, K]$ and the local time of $Z_{t}^{\pi}$ (see [3], Section 5.5 for a discussion in a simpler setting). We prefer here to avoid this problem by suitably restricting the class of admissible controls as specified in the following Definition. In what follows, the time scaling function $\gamma$ is assumed to be fixed.

Definition 2.6: A strategy $\pi$ is said to be admissible if the following conditions hold:

1) There exists a nonexploding solution to the martingale problem associated with (30), and the resulting process takes values in $(\epsilon, K)$ almost surely.

2) The process $\pi$ is bounded almost surely.

3) $J_{T}^{\gamma}(\pi)<+\infty$ almost surely.

We shall denote by $\mathcal{A}$ the set of admissible controls.

Our purpose is to find an admissible strategy $\pi^{*}$ that minimizes the ergodic cost function

$$
\lambda^{\pi, \gamma}=\limsup _{T \rightarrow+\infty} \frac{1}{T} E\left\{J_{T}^{\gamma}(\pi)\right\},
$$

i.e. that it is optimal in the mean and at the same time is also pathwise optimal in the sense of Theorem 2.4. We shall see, in particular, that the structure of the HJB equation makes the optimal feedback independent of the choice of $\gamma$. Moreover, for a special choice of $\gamma$, we shall see (see Theorem 3.6 below) that the optimal feedback is indeed an admissible strategy in the sense of the above Definition 2.6 and that it has all desired optimality properties.

\section{OPTIMAL SOLUTION}

\section{A. Solution for the criterion of optimality in the mean}

Consider the cost functional in (3) or (32) that is of the form of the general infinite-horizon average cost functional

$$
\lambda^{\pi}(z)=\limsup _{T \rightarrow \infty} \frac{1}{T} E^{\pi, z}\left\{\int_{0}^{T} c\left(Z_{t}^{\pi}, \pi_{t}\right) d t\right\}
$$

where $z$ is the initial value of $Z_{t}^{\pi}$ and $\pi$ is an admissible control i.e. such that

$$
\int_{0}^{T} c\left(Z_{t}^{\pi}, \pi_{t}\right) d t<\infty \quad \text { a.s. } \forall T
$$

Defining

$$
\lambda^{*}:=\inf _{\pi \in \mathcal{A}}\left\{\lambda^{\pi}(z), \forall z\right\}
$$


the general problem is to find $\pi^{*}$ such that $\lambda^{\pi^{*}}(z)=$ $\lambda^{*}$ for all $z$.

The main tool for solving this problem is based on the stationary HJB equation, namely on the following verification theorem. Its simple proof follows the argument sketched in [4], Sections 11.2.5 and 11.3.4. All steps are rigorously justified by the fact that the value function $\phi$ is uniformly bounded along the trajectories of $Z_{t}^{\pi}$ (by definition of admissible strategy).

Theorem 3.1: Given $Z_{t}^{\pi}$ satisfying (30), $\pi \in$ $\mathcal{A}$, and an instantaneous cost function $c(z, \pi)$, let $(\phi, \lambda)$ with $\phi \in \mathcal{C}^{2}$ be such that

$$
\inf _{\pi}\left[\mathcal{L}^{\pi} \phi(z)+c(z, \pi)\right]=\lambda
$$

where $\mathcal{L}^{\pi}$ is the generator of $Z_{t}^{\pi}$. Then, with $J_{T}(\pi):=\int_{0}^{T} c\left(Z_{t}^{\pi}, \pi_{t}\right) d t$,

i) $\quad \limsup _{T \rightarrow \infty} \frac{1}{T} E^{\pi}\left\{J_{T}(\pi)\right\} \geq \lambda$

ii) $\quad$ if $\pi(z):=\operatorname{argmin}_{\pi}\left[\mathcal{L}^{\pi} \phi(z)+c(z, \pi)\right] \mathrm{de}-$ fines an admissible control $\pi_{t}^{*}=\pi\left(z_{t}\right)$, then

$$
\begin{aligned}
& \lambda=\limsup _{T \rightarrow \infty} \frac{1}{T} E^{\pi^{*}}\left\{J_{T}\left(\pi^{*}\right)\right\} \text { i.e. } \\
& \lambda=\min _{\pi}\left\{\limsup _{T \rightarrow \infty} \frac{1}{T} E^{\pi}\left\{J_{T}(\pi)\right\}\right\}
\end{aligned}
$$

We shall now apply the above Theorem 3.1 to obtain a solution, optimal in the mean, for the specific case of our problem as described in the previous section II.

For this purpose put

$$
\eta=\frac{1}{2} \hat{\mu}^{\prime}\left(\sigma \sigma^{\prime}\right)^{-1} \hat{\mu} \quad, \quad \delta=\hat{r}+b^{\prime} \sigma^{-1} \hat{\mu}
$$

We shall make the following assumption

$$
\text { Assumption 3.2: i) } \quad \beta^{2}-2 \eta+2 \delta<0
$$

ii) $\quad 2 \eta-\delta \neq 0$

Remark 3.3: The somewhat restrictive condition i) above is introduced to make sure that, for the cost $c(z)=(z-1)^{2}$, the corresponding solution $(\phi, \lambda)$ of (36) is such that $\phi$ is a convex polynomial, which allows explicit computation of the optimal control. A more complete discussion on this point is given in Proposition 3.8.

Assume then also, for the moment, that for a given function $\gamma(z)$ there exists a triple $(\phi(z), \lambda, c(z))$, where $\phi(z) \in \mathcal{C}^{2}$, is bounded and has $\phi_{z z}(z)>0$, that satisfies, for $z \in(\epsilon, K)$,

$$
\begin{aligned}
E(z) & :=-\eta \gamma^{2}(z) \frac{\phi_{z}^{2}(z)}{\phi_{z z}(z)}+\delta z \gamma^{2}(z) \phi_{z}(z) \\
& +\frac{1}{2} \beta^{2} z^{2} \gamma^{2}(z) \phi_{z z}(z)+c(z)-\lambda=0
\end{aligned}
$$

Generalizing a procedure followed in [2], put

$$
\begin{aligned}
& Q(\pi ; z)=\frac{1}{2} \widetilde{\sigma}^{\pi} z^{2} \gamma^{2}(z) \phi_{z z}(z) \\
& \quad+z \gamma^{2}(z) \phi_{z}(z)\left(\pi^{\prime} \hat{\mu}+\hat{r}\right)+c(z)-\lambda
\end{aligned}
$$

where we use the shorthand notation

$$
\tilde{\sigma}^{\pi}=\left[\left(\sigma^{\prime} \pi-b\right)^{\prime}\left(\sigma^{\prime} \pi-b\right)+\beta^{2}\right] .
$$

We then have immediately

Lemma 3.4: For the model of section II the stationary Hamilton-Jacobi-Bellman (SHJB) equation

$$
\inf _{\pi \in \mathcal{A}}\left[\mathcal{L}^{\pi} \Phi+c\right]=\lambda
$$

is equivalent to

We also have

$$
\inf _{\pi \in \mathcal{A}}[Q(\pi ; z)]=0
$$

Lemma 3.5: Under the above assumptions on $\phi(z)$ i.e. $\phi(z) \in \mathcal{C}^{2}$ bounded and convex and satisfying (38), we have that, for fixed $z$, the function $Q(\pi ; z)$ in (38) is a non-negative definite quadratic form in $\pi$. Furthermore, letting

$$
\pi^{*}=-\left(\sigma \sigma^{\prime}\right)^{-1} \hat{\mu}\left(\frac{\phi_{z}(z)}{z \phi_{z z}(z)}\right)+\left(\sigma^{-1}\right)^{\prime} b
$$

one has

$$
\begin{gathered}
Q\left(\pi^{*} ; z\right)=-\eta \frac{\phi_{z}^{2}(z)}{\phi_{z z}(z)} \gamma^{2}(z)+\delta z \gamma^{2}(z) \phi_{z}(z) \\
+\frac{1}{2} \beta^{2} \phi_{z z}(z) z^{2} \gamma^{2}(z)+c(z)-\lambda=0
\end{gathered}
$$

Proof: For $z$ fixed, $Q(\cdot, z)$ is by definition a quadratic form in $\pi$. Furthermore, since $Q_{\pi \pi}=$ $z^{2} \gamma^{2}(z) \phi_{z z}(z)\left(\sigma \sigma^{\prime}\right)$ with $\sigma \sigma^{\prime}$ positive definite and $\phi$ convex, one has $Q \geq 0$.

To verify the second part of the statement, compute first the coefficients $\widetilde{\sigma}^{\pi}$ and $\left(\pi^{\prime} \hat{\mu}+\hat{r}\right)$ for $\pi=\pi^{*}$. 
Putting for simplicity $\alpha=\frac{\phi_{z}}{\phi_{z z}} \frac{1}{z}$ one obtains $\sigma^{\prime} \pi^{*}=$ $-\alpha \sigma^{-1} \hat{\mu}+b$ from which

$$
\left[\sigma^{\prime} \pi^{*}-b\right]^{\prime}\left[\sigma^{\prime} \pi^{*}-b\right]=\alpha^{2} \hat{\mu}^{\prime}\left(\sigma \sigma^{\prime}\right)^{-1} \hat{\mu}=2 \alpha^{2} \eta
$$

and thus

$$
\widetilde{\sigma}^{\pi^{*}}=2 \alpha^{2} \eta+\beta^{2}
$$

Furthermore,

$$
\begin{aligned}
\left(\pi^{*}\right)^{\prime} \hat{\mu}+\hat{r} & =-\alpha \hat{\mu}^{\prime}\left(\sigma \sigma^{\prime}\right)^{-1} \hat{\mu}+b^{\prime}\left(\sigma^{-1}\right) \hat{\mu}+\hat{r} \\
& =-2 \alpha \eta+\delta
\end{aligned}
$$

Substituting these expressions in (38) one obtains

$$
\begin{gathered}
\frac{1}{2} \phi_{z z} z^{2} \gamma^{2} 2 \alpha^{2} \eta+\frac{1}{2} \phi_{z z} z^{2} \gamma^{2} \beta^{2} \\
+\phi_{z} z \gamma^{2}(\delta-2 \alpha \eta)+c-\lambda \\
=z^{2} \gamma^{2} \phi_{z z}\left[\frac{\phi_{z}^{2}}{\phi_{z z}^{2}} \frac{1}{z^{2}}\right] \eta+\frac{1}{2} \phi_{z z} z^{2} \gamma^{2} \beta^{2}+\phi_{z} z \gamma^{2} \delta \\
-\phi_{z} z \gamma^{2}\left(\frac{2 \phi_{z}}{\phi_{z z}} \frac{1}{z} \eta\right)+c-\lambda
\end{gathered}
$$

namely

$$
\begin{aligned}
Q\left(\pi^{*} ; z\right)= & -\eta \frac{\phi_{z}^{2}(z)}{\phi_{z z}(z)} \gamma^{2}(z)+\delta z \gamma^{2}(z) \phi_{z}(z) \\
& +\frac{1}{2} \beta^{2} z^{2} \gamma^{2}(z) \phi_{z z}(z)+c(z)-\lambda
\end{aligned}
$$

By the assumption that the pair $(\phi, \lambda)$ satisfies (38) this then leads to

$$
Q\left(\pi^{*} ; z\right)=0, \quad \forall z \in(\epsilon, K)
$$

In the next Theorem we make a special choice for the function $\gamma(z)$ and assume that $\phi(z)$ is a second order convex polynomial; such a $\phi$ will in fact be shown below (see Proposition 3.8 and Corollary 3.12) to be a solution to (38) when $c(z)$ is a second-order polynomial and this independently of the choice of $\gamma(z)$.

Theorem 3.6: Put

$$
\gamma(z)=\frac{(K-z)(z-\epsilon)}{(K-\epsilon) z}
$$

and assume that $\phi(z)=A z^{2}+B z+C$ is, for suitable constants $A>0, B, C$, a solution of (38) corresponding to the given $\gamma(z)$ and a chosen cost function $c(z)$, bounded from below. Then the control $\pi^{*}$ in (42) has the property that

a) it is admissible in the sense of Definition 2.6

b) it satisfies

$$
\pi^{*}=\arg \inf _{\pi}\left[\mathcal{L}^{\pi} \Phi+c\right]
$$

and is optimal in the mean for the problem with state variable $Z_{t}^{\pi, \gamma}$ and with the ergodic cost functional (32).

Remark 3.7: The optimal control $\pi^{*}$ in (42) does not depend directly on the chosen $\gamma(z)$; it does not even depend indirectly on $\gamma$ since the solution $\phi$ of (38) as well will be shown in III-A.2 to be independent of $\gamma$.

Proof of the Theorem : To prove a) it suffices to show that, for the given choice of $\gamma(z)$ in (44) and for $\pi=\pi^{*}$, equation (30) has a nonexploding solution $Z_{t}^{\pi^{*}, \gamma} \in[\epsilon, K]$. For this purpose assume for the moment that $Z_{t}^{\pi^{*}, \gamma} \in(\epsilon, K)$ and define

$$
\Psi_{t}=\Psi\left(Z_{t}\right):=\log \left(\frac{Z_{t}-\epsilon}{K-Z_{t}}\right)
$$

If, after applying Ito's formula, it can be shown that $\Psi_{t}$ is the unique strong solution of the corresponding equation, then one has shown that, indeed,

$$
Z_{t}:=Z\left(\Psi_{t}\right)=\frac{K e^{\Psi_{t}}+\epsilon}{1+e^{\Psi_{t}}} \in(\epsilon, K)
$$

To this effect replace $\pi$ by $\pi^{*}$ from (42) in (30). Recalling the definitions of $\eta$ and $\delta$ in (37) one then has

$$
\begin{aligned}
d Z_{t}=Z_{t}[ & \left(\delta-2 \eta-\eta \frac{B}{A} \frac{1}{Z_{t}}\right) \gamma^{2} d t \\
& \left.\quad+\left(-\frac{\hat{\mu}}{\sigma}-\frac{\hat{\mu}}{\sigma} \frac{B}{2 A} \frac{1}{Z_{t}}\right) \gamma d w_{t}-\beta \gamma d v_{t}\right] \\
=Z_{t}[ & \left(C_{1}+C_{2} \frac{1}{Z_{t}}\right) \gamma^{2} d t \\
& \left.\quad+\left(C_{3}+C_{4} \frac{1}{Z_{t}}\right) \gamma d w_{t}-\beta \gamma d v_{t}\right]
\end{aligned}
$$

with obvious meaning of the shorthand symbols $C_{1}, \cdots, C_{4}$. Putting

$$
\sigma_{\Psi}^{2}=Z^{2} \gamma^{2}\left[\left(C_{3}+C_{4} \frac{1}{Z}\right)^{2}+\beta^{2}\right]
$$


and noticing that

$$
\begin{aligned}
Z(\Psi) & =\frac{K e^{\Psi}+\epsilon}{1+e^{\Psi}} \\
\frac{\partial \Psi}{\partial Z} & =\frac{K-\epsilon}{(Z-\epsilon)(K-Z)}=\frac{1}{\gamma Z} \\
\frac{\partial^{2} \Psi}{\partial Z^{2}} & =\frac{(K-\epsilon)(2 Z-K-\epsilon)}{(Z-\epsilon)^{2}(K-Z)^{2}} \\
& =\frac{2 Z-K-\epsilon}{K-\epsilon} \frac{1}{(\gamma Z)^{2}}
\end{aligned}
$$

by applying Ito's rule we obtain for $\Psi_{t}$ the following equation

$$
\begin{aligned}
d \Psi= & \left\{\left[\left(C_{1}+C_{2} \frac{1}{Z}\right) \gamma(Z)\right.\right. \\
+ & \left.\frac{1}{2}\left(\left(C_{3}+C_{4} \frac{1}{Z}\right)^{2}+\beta^{2}\right) \frac{2 Z-K-\epsilon}{K-\epsilon}\right] d t \\
& \left.+\left(C_{3}+C_{4} \frac{1}{Z}\right) d w_{t}-\beta d v_{t}\right\}_{Z=Z(\Psi)}
\end{aligned}
$$

Since

$$
\begin{gathered}
\left.\gamma(Z)\right|_{Z=Z(\Psi)}=\frac{K-\epsilon}{K e^{\Psi}+\epsilon} \frac{e^{\Psi}}{1+e^{\Psi}} \\
\left.\frac{2 Z-K-\epsilon}{K-\epsilon}\right|_{Z=Z(\Psi)}=\frac{e^{\Psi}-1}{e^{\Psi}+1}
\end{gathered}
$$

we finally obtain

$$
\begin{aligned}
d \Psi= & {\left[C_{1} \frac{(K-\epsilon) e^{\Psi}}{\left(K e^{\Psi}+\epsilon\right)\left(1+e^{\Psi}\right)}\right.} \\
& +C_{2} \frac{(K-\epsilon) e^{\Psi}}{\left(K e^{\Psi}+\epsilon\right)^{2}} \\
& +\frac{C_{3}^{2}+\beta^{2}}{2} \frac{e^{\Psi}-1}{e^{\Psi}+1}+ \\
& +C_{3} C_{4} \frac{e^{\Psi}-1}{K e^{\Psi}+\epsilon} \\
& \left.+\frac{C_{4}^{2}}{2} \frac{\left(1+e^{\Psi}\right)\left(e^{\Psi}-1\right)}{\left(K e^{\Psi}+\epsilon\right)^{2}}\right] d t+ \\
& +\left[C_{3}+C_{4} \frac{1+e^{\Psi}}{K e^{\Psi}+\epsilon}\right] d w_{t}-\beta d v_{t}
\end{aligned}
$$

To verify the existence of a strong solution of this last equation (52), it suffices to show that the drift and volatility coefficients have a bounded derivative. For this purpose it suffices to notice that the derivatives of all the coefficients are ratios of polynomials in $e^{\Psi}$. All denominators are different from zero and so these functions are continuous. In addition it can be verified that the limits for $|\Psi| \rightarrow \infty$ are bounded implying that the functions themselves are bounded.
Coming to b) notice that by Lemma 3.4 the (SHJB) can be written as

$$
\inf _{\pi}[Q(\pi ; z)]=0
$$

By Lemma 3.5, $Q \geq 0$ and, in particular, $Q\left(\pi^{*} ; z\right)=$ 0 , so one has

$$
Q\left(\pi^{*} ; z\right)=\inf _{\pi}[Q(\pi ; z)]=0
$$

and therefore $\pi^{*}=\arg \inf _{\pi}[Q(\pi ; z)]$.

Furthermore, as will be shown below (see in particular Corollary 3.17) the triple $[(\phi, 0) ; c]$ solves (SHJB) for $c=c^{\epsilon, K}$ given in (69) below and so the feedback law $\pi^{*}$ is optimal in the mean.

We have now obtained a control that is optimal in the mean for the problem of section II under the assumption that there exists a triple $(\phi(z), \lambda, c(z))$, with $\phi(z)$ of the form $\phi(z)=A z^{2}+B z+C$, that satisfies (38). The latter is a nonlinear PDE of the kind arising in continuous-time stochastic control problems.

Next we shall thus study this equation (38) and for this purpose we shall associate with it a simpler equation, obtained from (38) by putting $\gamma(z) \equiv 1$, i.e.

$$
\begin{array}{r}
\tilde{E}(z):=-\eta \frac{\phi_{z}^{2}(z)}{\phi_{z z}(z)}+\delta z \phi_{z}(z)+\frac{1}{2} \beta^{2} z^{2} \phi_{z z}(z) \\
+c(z)-\lambda=0
\end{array}
$$

that we shall call the auxiliary equation; we shall look for solutions for all $z \in \mathbb{R}$. Below, when studying equations (38) and (53) we shall consider as solution an entire triple $(\phi, \lambda, c)$. In subsection III-A.2 we shall show that a solution $(\phi, \lambda, c)$ for (38) can be obtained rather straightforwardly, once a solution $(\tilde{\phi}, \tilde{\lambda}, \tilde{c})$ for (53) has been found and so we study first the auxiliary equation (53).

In general, the instantaneous cost function $c(z)$ is given and in our case, where the purpose is that of controlling $Z_{t}^{\pi}$ such that it stays close to 1 , a natural choice could be $c(z)=(z-1)^{2}$. Notice however that 
the same purpose can be achieved also with other cost functions provided that they have a minimal value in $z=1$. In fact, as will be shown in subsection IIIA.2 below, a convenient cost function that allows to obtain a solution $(\phi, \lambda, c)$ of (38) is more complex than the simple $c(z)=(z-1)^{2}$.

Coming then to (53), notice that it becomes a polynomial equation whenever $\phi(z)$ and $c(z)$ are polynomials. In the next subsection III-A.1 we shall thus study solutions $(\tilde{\phi}, \tilde{\lambda}, \tilde{c})$ of (53) under the assumption that $\tilde{c}$ is a polynomial, bounded from below and of which we shall then also require that it has minimal value in $z=1$. It turns out (see Proposition 3.8 and its Corollary 3.12) that the only such polynomial is $\tilde{c}(z)=(z-1)^{2}$ (the corresponding $c(z)$ to obtain a solution to (38) will however be different from $(z-1)^{2}$ as can be seen from Corollary 3.17 below).

1) Solution of the nonlinear PDE in the polynomial case: We have the following

Proposition 3.8: Let $c(z)$ be a polynomial function in $z$ of positive degree, i.e. $c(z)=\sum_{j=0}^{K} c_{j} z^{j}$ with $c_{K}>0$.

a) If $c$ is of degree 2 then, modulo a constant factor, there exists one solution $(\phi(z), \lambda)$ of (53) with $\phi(z)$ a polynomial if and only if either Assumption 3.2 ii) is satisfied $c_{1}=$ 0. If Assumption 3.2 is satisfied then the solution is unique, and is given by

$\phi(z)=A(z+B)^{2} \quad ; \quad \lambda=-2 A \eta B^{2}+c_{0}$

where

$$
\left\{\begin{array}{l}
A=\frac{-c_{2}}{\beta^{2}+2 \delta-2 \eta} \\
B=\frac{c_{1}}{2 A(2 \eta-\delta)}
\end{array}\right.
$$

If Assumption 3.2 is not satisfied and $c_{1}=$ 0 then the solutions are still given by (54), with $A$ as in (55) and $B$ generic. In both cases $\phi$ is convex if and only if assumption 3.2 i) hold

b) If $c$ is of degree larger than 2, then there exists a solution $(\phi(z), \lambda)$ of (53), with $\phi(z)$ a polynomial, for an open set of values for the triple of parameters $(\eta, \delta, \beta)$ if and only if $c(z)$ has the form

$$
c(z)=c_{K} z^{K}+c_{0} .
$$

In this case the solution is unique and it is given by

$$
\begin{aligned}
\phi(z) & =\frac{-c_{K}}{\frac{\beta^{2}}{2} K(K-1)+\delta K-\eta \frac{K}{K-1}} z^{K} \\
\lambda & =c_{0}
\end{aligned}
$$

Moreover $\phi$ is convex if and only if $K$ is even and the condition, that strengthen 3.2 i),

$$
\beta^{2}(K-1)^{2}+2 \delta(K-1)-2 \eta<0
$$

holds.

Remark 3.9: The main consequence of Proposition 3.8 is that polynomials of the form $c(z)=$ $c_{1}(z-1)^{2}+c_{2}$ are the only polynomials attaining the minimum value at $z=1$ and such that there exists a corresponding solution $(\phi(z), \lambda)$ of (53) with $\phi(z)$ a convex polynomial.

Remark 3.10: We obtained a solution where $\phi(z)$ is a convex polynomial. No claim is made that this is the only possible solution. We were aiming at obtaining a computable solution and for this purpose we did not fix a priori a cost function $c(z)$. Since a same objective can be achieved with different cost functions, we were rather looking for a suitable triple $(c(z), \phi(z), \lambda)$ with a meaningful $c(z)$ that leads to a solution independently of the choice of the time transformation. In so doing, it turned out that a second order polynomial $c(z)$ with ensuing polynomial $\phi(z)$ allowed us to achieve this objective.

Remark 3.11: The requirement that a polynomial solution exists for an open set of values for 
the triple of parameters $(\eta, \delta, \beta)$ is rather natural. Indeed, in real application, model parameters are only approximately identified, so that one seeks for results that holds for "generic" values of the parameters.

Proof of Proposition 3.8: Since we consider only polynomial $\phi(z)$, its degree must not be less than 2 in order for the denominator in (53) to be different from zero. Assuming than that there exists a solution to (53) with $\operatorname{deg}(\phi) \geq 2$, all the terms after the first one in (53) are polynomials implying that also the first term (in $\eta$ ) has to be a polynomial, which is equivalent to requiring that $\phi_{z z}$ divides $\phi_{z}^{2}$. This is immediately seen to be true if $\phi^{\prime}(z)$ is of the simple form $\phi^{\prime}(z)=k(z-\beta)^{\alpha}$. Putting $f(z):=$ $\phi^{\prime}(z)$ let us then consider general polynomials

$$
f(z)=\prod_{i=1}^{n}\left(z-\beta_{i}\right)^{\alpha_{i}}, \quad \beta_{i} \neq \beta_{j} \text { if } i \neq j,
$$

where the $\beta_{i}$ are possibly complex. The ratio $\frac{f^{2}}{f^{\prime}}$ can be written as $\frac{f}{D(\log f)}$ where

$$
\log f=\log \sum_{i=1}^{n} \alpha_{i} \log \left(z-\beta_{i}\right)
$$

Therefore,

$$
D \log f=\sum_{i} \frac{\alpha_{i}}{z-\beta_{i}}=\frac{\sum_{i} \alpha_{i} \prod_{j \neq i}\left(z-\beta_{j}\right)}{\prod_{i}\left(z-\beta_{i}\right)}
$$

and so

$$
\frac{f^{2}}{f^{\prime}}=\frac{\prod_{i}\left(z-\beta_{i}\right)^{\alpha_{i}+1}}{\sum_{i} \alpha_{i} \prod_{j \neq i}\left(z-\beta_{j}\right)}
$$

It is immediately seen that, since $\beta_{i} \neq \beta_{j}$, none of the zeroes of the numerator, namely $\beta_{j}, j=1, \ldots, n$, causes the denominator to vanish, but then $f^{\prime}$ cannot divide $f^{2}$.

The only possibility that remains is therefore $f=$ $k(z-\beta)^{\alpha}$.

In this case, modulo additive constants that are irrelevant for equation (53),

$$
\phi(z)=\frac{k}{\alpha+1}(z-\beta)^{\alpha+1}
$$

In other words, $\phi$ must be of the form

$$
\phi(z)=A(z+B)^{K} .
$$

It is easily seen that with such a $\phi$ of generic degree $K \geq 2$, the first term in (53) is a polynomial of degree $K$ and therefore, necessarily, $\operatorname{deg}(c)=K(\lambda$ is a constant). This implies

$$
\operatorname{deg}(\phi)=\operatorname{deg}(c)
$$

After these preliminary considerations we can now analyze individually the two cases of $\operatorname{deg}(c)=2$ and $\operatorname{deg}(c)>2$.

Case a) : With

$c(z)=c_{2} z^{2}+c_{1} z+c_{0} \quad$ and $\quad \phi(z)=A(z+B)^{2}$ equation (53) becomes

$$
-2 A \eta(z+B)^{2}+\delta 2 z A(z+B)+\beta^{2} z^{2} A+c(z)-\lambda=0 .
$$

This expression has to vanish for all values of $z$ and so

$$
\left\{\begin{array}{l}
-2 \eta A+2 \delta A+\beta^{2} A+c_{2}=0 \\
-4 \eta A B+2 \delta A B+c_{1}=0 \\
-2 \eta A B^{2}+c_{0}-\lambda=0
\end{array}\right.
$$

from where all claims concerning the case $\operatorname{deg}(c)=$ 2 easily follow.

Case b) : With

$$
c(z)=\sum_{j=0}^{K} c_{j} z^{j} \quad \text { and } \quad \phi=A(z+B)^{K}
$$

equation (53) becomes

$$
\begin{aligned}
& -\eta A \frac{K}{K-1}(z+B)^{K}+\delta A K z(z+B)^{K-1} \\
& +\frac{\beta^{2} A K(K-1)}{2} z^{2}(z+B)^{K-2}+c-\lambda=0
\end{aligned}
$$

It holds only if all the coefficients vanish.

Consider first this condition for the coefficient of the term with highest degree

$$
-\eta \frac{K}{K-1} A+\delta K A+\frac{\beta^{2}}{2} K(K-1) A+c_{K}=0
$$

from which we obtain

$$
A=\frac{-c_{K}}{\frac{\beta^{2}}{2} K(K-1)+\delta K-\eta \frac{K}{K-1}}
$$


Concerning the other terms, consider the condition for the coefficient of the generic $j$-th term with $j>0$

$$
\begin{gathered}
-\eta A \frac{K}{K-1}\left(\begin{array}{c}
K \\
j
\end{array}\right) B^{K-j}+\delta A K\left(\begin{array}{c}
K-1 \\
j-1
\end{array}\right) B^{K-j} \\
+\frac{\beta^{2}}{2} A K(K-1)\left(\begin{array}{c}
K-2 \\
j-2
\end{array}\right) B^{K-j}+c_{j}=0
\end{gathered}
$$

Since we know already the value of $A$, from this equation we obtain for $B$

$$
\begin{aligned}
B= & \left(-c_{j}\left\{A \left[-\eta \frac{K}{K-1}\left(\begin{array}{c}
K \\
j
\end{array}\right)+\delta K\left(\begin{array}{c}
K-1 \\
j-1
\end{array}\right)\right.\right.\right. \\
& \left.\left.\left.+\frac{\beta^{2}}{2} K(K-1)\left(\begin{array}{c}
K-2 \\
j-2
\end{array}\right)\right]\right\}^{-1}\right)^{\frac{1}{K-j}}
\end{aligned}
$$

for every $j=1, \ldots, K-1$. Note that if one $c_{j}=0$, the $B=0$. Otherwise the right hand side of (60) must be independent of $j$. This fact can be expressed by a system of polynomial equations for $(\eta, \delta, \beta)$ that are not identities, and that, therefore, cannot be satisfied in an open set. Thus, the only possibility is that $B=$ 0 and $c_{j}=0$ for $j=1, \ldots, K-1$.

Finally, for the constant term there remains the equation $c_{0}-\lambda=0$.

To conclude, for the case when $\operatorname{deg}(c)>2$ one can find a solution of (53) and thus of (SHJB) only if $c(z)=c_{K} z^{K}+c_{0}$ and the corresponding solution is given by the pair $(\phi, \lambda)$ in (57).

This Proposition leads immediately to

Corollary 3.12: Given the instantaneous cost function $c(z)=(z-1)^{2}$, under Assumption 3.2 there exists one and only one solution $(\phi, \lambda)$ to (53) with $\phi(z)$ a polynomial function. More precisely one has $\phi(z)=(p z+q)^{2}$ with

$$
\left\{\begin{array}{l}
p=\sqrt{-\left(\beta^{2}+2 \delta-2 \eta\right)^{-1}} \\
q=\frac{\sqrt{-\left(\beta^{2}+2 \delta-2 \eta\right)}}{2 \eta-\delta}
\end{array}\right.
$$

Furthermore,

$$
\lambda=2 \eta \frac{\beta^{2}+2 \delta-2 \eta}{(2 \eta-\delta)^{2}}+1=\frac{\delta^{2}+2 \eta \beta^{2}}{(\delta-2 \eta)^{2}}
$$

Remark 3.13: The result of Corollary 3.12, combined with Lemma 3.16 below, justifies the assumption made in Theorem 3.6 that there exists $\phi(z)$ of the form $\phi(z)=A z^{2}+B z+C$ that satisfies (38).

2) Methodology for determining a control that is optimal in the mean: The main purpose of this subsection is to show how from a solution of (53) one obtains a solution of (38) with $\phi(z)=A z^{2}+$ $B z+C$ for suitable values of $A>0, B, C$ and that corresponds to an instantaneous cost function $c(z)$ having minimal value in $z=1$.

We start with the following immediate

Lemma 3.14: If the triple $(\phi, \lambda, c)$ satisfies (38), the same is true for $(\phi, \tilde{\lambda}, \tilde{c})$ with $\tilde{\lambda}=\lambda+\zeta, \tilde{c}=$ $c+\zeta$ for any real value $\zeta$.

Remark 3.15: This Lemma allows us to translate the values of $c$ which in turn allows one to obtain the most convenient problem setup. In particular, one may choose $\tilde{c}=c-\lambda$ so that $\tilde{\lambda}=0$ for which (SHJB) in (40) becomes

$$
\inf _{\pi \in \mathcal{A}}\left[\mathcal{L}^{\pi} \Phi+\tilde{c}\right]=0
$$

Next we prove

Lemma 3.16: Given the dynamics of $Z_{t}^{\pi}$ as in (30), where $\gamma(z)$ is any sufficiently regular function of $z$, let the instantaneous cost function $c(z)$ have the form

$$
c(z)=\gamma^{2}(z)[C(z)-\Lambda]
$$

where $C(z)$ is a given function of $z$ and $\Lambda$ a suitable costant. Then the triple

$$
[(\phi(z), 0) ; c(z)]
$$

solves (38) if and only if the triple

$$
[(\phi, \Lambda) ; C(z)]
$$

solves the auxiliary equation (53).

Proof: "only if" Suppose that $[(\phi(z), 0) ; c(z)]$ solves (38); it means that we may rewrite this equa- 
tion as :

$$
\begin{aligned}
& -\eta \gamma^{2}(z) \frac{\phi_{z}^{2}(z)}{\phi_{z z}(z)}+\delta z \gamma^{2}(z) \phi_{z}(z) \\
+ & \frac{1}{2} \beta^{2} z^{2} \gamma^{2}(z) \phi_{z z}(z)+\gamma^{2} z[C(z)-\Lambda]=0 .
\end{aligned}
$$

Dividing by $\gamma^{2}$ it follows immediately that $[(\phi, \Lambda) ; C(z)]$ solves the auxiliary equation (53).

The "if" part is completely analogous : if the triple $[(\phi, \Lambda) ; C(z)]$ solves

$$
-\eta \frac{\phi_{z}^{2}(z)}{\phi_{z z}(z)}+\delta z \phi_{z}(z)+\frac{1}{2} \beta^{2} z^{2} \phi_{z z}(z)+c(z)=\lambda
$$

then, bringing $\lambda=\Lambda$ on the left of the equal sign and multiplying by $\gamma^{2}$, one obtains the statement.

If for our control problem with controlled state variable $Z_{t}^{\pi}$ as in (30) we have an instantaneous cost function of the from as in (64), then Lemma 3.16 allows us to search for a triple $(\phi, \lambda, c)$ that solves (38), by solving (53), i.e. by putting $\gamma(z) \equiv 1$. Notice that we are allowed to do this only at the level of the algorithm to obtain a solution to the given problem. The problem itself looses its meaning for $\gamma(z) \equiv 1$ since then the process $Z_{t}^{\pi}$ does not anymore possess the required ergodic properties as pointed out in section II-A.

In what follows, we fix the time-scaling function $\gamma$ according to (44), and, consequently, the instantaneous cost according to (64). More precisely, the instantaneous cost for the time scaled process is given by

$$
c^{\epsilon, K}(z)=\frac{(K-z)^{2}(z-\epsilon)^{2}}{(K-\epsilon)^{2} z^{2}}\left[(z-1)^{2}-\Lambda\right]
$$

where

$$
\Lambda=\frac{\delta^{2}+2 \eta \beta^{2}}{(\delta-2 \eta)^{2}}>0
$$

with $\eta$ and $\delta$ as given in (37).

Corollary 3.17: Given $c^{\epsilon, K}(z)$ as in (69), one has that the pair $(\phi, 0)$, with $\phi$ as in Corollary 3.12, solves (38).

Proof: By Corollary 3.12 we have that $(\phi, \Lambda=$ $\lambda)$ with $C(z)=(z-1)^{2}$ solves the auxiliary equation
(53). By Lemma 3.16 the triple $\left[(\phi(z), 0) ; c^{\epsilon, K}(z)\right]$ then solves (38).

We come now to the main theorem concerning the control that is optimal in the mean.

Theorem 3.18: Given the control problem with controlled process $Z_{t}^{\pi}$ as in (30) where $\gamma(z)$ is chosen according to (44), the criterion is the ergodic cost functional (32), and the instantaneous cost function is a $c^{\epsilon, K}(z)$ according to (69). Then, under assumption 3.2 , the control

$$
\begin{aligned}
\pi_{t}^{*}=-\left(\sigma \sigma^{\prime}\right)^{-1} \hat{\mu} & \frac{\left(2 \eta-2 \delta-\beta^{2}\right)}{(2 \eta-\delta)} \frac{1}{z_{t}} \\
& +\left(\sigma^{-1}\right)^{\prime} b-\left(\sigma \sigma^{\prime}\right)^{-1} \hat{\mu}
\end{aligned}
$$

is optimal in the mean.

Proof: By Corollary 3.17 we have that a solution to $(38)$ is $\left(\phi(z), 0, c^{\epsilon, K}(z)\right)$, where $c^{\epsilon, K}(z)$ is as in (44) and $\phi(z)$ as in Corollary 3.12 namely of the form as required in Theorem 3.6 with

$$
\left\{\begin{array}{l}
A=p^{2}=\left(2 \eta-2 \delta-\beta^{2}\right)^{-1} \\
B=2 p q=2(2 \eta-\delta)^{-1} \\
C=q^{2}=\frac{2 \eta-2 \delta-\beta^{2}}{(2 \eta-\delta)^{2}}
\end{array}\right.
$$

Since the form assumed here for the function $\gamma(z)$ is the same as that required in Theorem 3.6, we have that $\pi^{*}$ in (42) is an optimal control where we can now replace $\phi_{z}(z)$ and $\phi_{z z}(z)$ by their expression resulting from $\phi(z)=A z^{2}+B z+C$ with $A, B, C$ as in (72). Notice that, by assumption 3.2, we have $A>$ 0 as required. This then leads to (71) thus proving the Theorem.

To conclude, notice that the triple $(\phi, \lambda, c)$ that solves (38) in correspondence of $c=c^{\epsilon, K}$ and $\phi(z)=A z^{2}+B z+C$ has $\lambda=0$. There is no contradiction in the fact that the optimal value for the control problem is zero, since, with the translation by $\Lambda$, the instantaneous cost function can take positive as well as negative values. 
B. Solution for the criterion of pathwise optimality

In this final section, we show pathwise opimality of the control $\pi^{*}$ given in (71). The explicit knowledge of the solution of (SHJB) makes pathwise optimality an almost immediate application of Theorem 2.4.

Theorem 3.19: Under the assumptions of Theorem 3.18, the control in (71) is $g$-optimal a.s. for every $g(\cdot)$ satisfying condition (iii) in Theorem 2.4.

Proof: We have to verify that conditions (i) and (ii) in Theorem 2.4 hold. For Condition (i), observe that $\phi$ is a polynomial of degree two, and the diffusion coefficient in the state equation (30) is

$$
\left(\gamma(z) z\left(\pi^{\prime} \sigma-b^{\prime}\right), \gamma(z) z\right) .
$$

Since both state and control are assumed to be bounded, Condition (i) follows.

Similarly, Condition (ii) follows from the fact that the instantaneous cost function $\gamma^{2}(z)(c(z)-\Lambda)$ is continuous in $z$.

\section{REFERENCES}

[1] K. Ito, H.P. McKean, Jr, Diffusion Processes and their Sample Paths, Springer-Verlag, Berlin, 1974.

[2] S. Browne, Beating a moving target : Optimal portfolio strategies for outperforming a stochastic benchmark, Finance and Stochastics 3 (1999), pp. 275-294.

[3] I. Karatzas, S.E. Shreve, Brownian Motion and Stochastic Calculus, Springer-Verlag, New York, 1988.

[4] H. Kushner, Introduction to Stochastic Control, Holt, Rinehart and Winston, Inc., 1971.

[5] R.Merton, Optimum Consumption and Portfolio Rules in a Continuous Time model, J. Econ. Theory 3 (1971), pp.373413.

[6] È. L. Presman, Optimality almost surely and in probability for a stochastic linear-quadratic regulator, Theory Probab. Appl., 42 (1997), pp. 531-535.

[7] È. L. Presman, V. I. Rotar, and M. Taksar, Optimality in probability and almost surely. The general scheme and a linear quadratic regulator problem, Stochastics Stochastic Rep., 43 (1993), pp. 127-137.

[8] V. I. Rotar, Connectivity property and optimality almost surely and in probability, in New Trends in Probability and Statistics, VSP, Utrecht, 1991, pp. 528-539.
[9] P. Dai Pra, G.B. Di Masi, and B. Trivellato, Pathwise optimality in stochastic control, SIAM J. Control Optim. 39 (2000), no. 5, 1540-1557

[10] M. Tolotti, Strategie di portafoglio ottimali per il "tracking" di un indice, Laurea thesis, University of Padova, 2002. 